\title{
2057 Quantitative estimation of right ventricular function obtained from images acquired in oblique sagittal orientation is accurate and easier compared to that obtained by the left ventricular short axis and oblique transverse orientations
}

\author{
Benjamin Cheong*, Preethi Nambi, Mercedes Pereyra and Raja Muthupillai
}

Address: St. Luke's Episcopal Hospital, Department of Radiology, Houston, TX, USA

* Corresponding author

from I th Annual SCMR Scientific Sessions

Los Angeles, CA, USA. I-3 February 2008

Published: 22 October 2008

Journal of Cardiovascular Magnetic Resonance 2008, I0(Suppl I):A326 doi:I0.I I86/I532-429X-I0-SI-A326

This abstract is available from: http://jcmr-online.com/content/I0/SI/A326

(c) 2008 Cheong et al; licensee BioMed Central Ltd.

\section{Introduction}

In clinical practice, $\mathrm{RV}$ volumes are estimated from the same short-axis imaging orientations (LVSAX) used to estimate LV volumes. However, estimating RV volumes using images acquired in the LVSAX orientation is often challenging. The crescentic shape of the RV near the base of the heart makes it difficult to delineate RV chamber due to the through plane motion of the tricuspid valve (TV) and the pulmonic valve (PV) during the cardiac cycle. The purpose of this study is to test if an alternate imaging orientation would provide better visualization of the RV valves, and provide a robust quantitation of $\mathrm{RV}$ volumes.

\section{Purpose}

The purpose of this study is to compare the RV functional metrics obtained from cine steady-state free precession (SSFP) images acquired in three different imaging orientations.

\section{Methods}

Twenty healthy volunteers (12 males; age: $37 \pm 9$ years) with no past cardiac history were imaged on a $1.5 \mathrm{~T}$ commercial scanner. A set of contiguous slices covering the ventricular chambers of the heart were obtained using a SSFP sequence with the following parameters: TR/TE/flip: 3.2/1.6/65 ; acquired resolution: $2.25 \times 2.5 \times 8 \mathrm{~mm}$, temporal resolution: $40-50 \mathrm{msec}$; scan time: $5-6 \mathrm{sec} / \mathrm{slice}$. The imaging orientations are as follow: (1) LVSAX: Conventional double oblique short axis left-ventricular short axis orientation; (2) RVSAG: A set of contiguous slices acquired parallel to the long axis of the RV defined by the line intersecting the center of TV annulus and the RV apex. This orientation enables visualizing both the TV, and the PV in the plane of imaging; (3) RVAX: A set of contiguous slices that are oriented perpendicular to the line connecting the center of the PV annulus to the juncture of the RV free wall and the inferior wall.

In addition, flow through the aorta was measured using a phase contrast MR imaging method. A single blinded observer measured the RV volumes from SSFP images along all three imaging orientations using a commercially available workstation (ViewForum, Philips Medical Systems). An independent observer also ranked the increasing ease with which the PV and TV were visualized in images on a score of 1 through 3 .

\section{Results}

The end-diastolic (EDV), end-systolic (ESV), stroke-volume (SV), and ejection fraction (EF) were analyzed for a total of 60 ventricular volumes (20 subjects, 3 orientations per subject).

The RVSAG orientation required the fewest number of slices $(8.7 \pm 1.2)$ compared to LVSAX $(11.6 \pm 1.9)$ or RVAX $(12.6 \pm 1.7)$ to cover the entire RV and this difference was statistically significant $(\mathrm{p}<0.0001)$. TV and PV were better 
visualized on RVSAG orientation than on the LVSAX or RVAX orientations $(\mathrm{p}<0.05)$.

One way ANOVA showed that there was no statistically significant difference in the estimation of EDV, ESV, SV or $\mathrm{EF}$ between the three imaging orientations. While SV measured from images obtained all three orientations correlated well with $\left(\mathrm{r}^{2}>0.9\right)$ aortic quantitative flow measurements, the RVSAG orientation yielded the highest correlation. Bland-Altman analysis reveals that RV functional metrics obtained from RVSAG orientation had the least intra-observer variability.

\section{Conclusion}

Quantifying RV function in the RVSAG orientation offers the following advantages: (1) Provide RV functional metrics that are comparable to more traditional LVSAX or RVAX orientations; (2) Clear delineation of both PV and TV both during diastole and systole resulting in improved reader confidence for circumscribing the RV; (3) Fewer slices are required to cover the entire RV; and (4) Lower intra-observer variability in the estimation of quantitative functional values describing RV function. RV function is better assessed from images acquired in oblique sagittal orientation (RVSAG) compared to conventional orientations. RVSAG orientation requires fewer slices, better delineation of both pulmonic and triscuspid valves, and quantifies RV function accurately. 